\title{
Adrenomedullin Stimulates Porcine Sinus Node Automaticity
}

\section{Stenberg TA ${ }^{1,2}$, Koren $\mathbf{S}^{1}$, Kildal $\mathrm{AB}^{1,3}$ and Myrmel $\mathrm{T}^{1,4 *}$}

${ }^{1}$ Department of Clinical Medicine, UiT The Arctic University of Norway, Tromsø, Norway

${ }^{2}$ Department of Urology and Endocrine Surgery, University Hospital of North Norway, Tromsø, Norway

${ }^{3}$ Department of Anesthesiology, University Hospital of North Norway, Tromsø, Norway

${ }^{4}$ The Heart and Lung Clinic, University Hospital of North Norway, Tromsø, Norway

\begin{abstract}
Adrenomedullin (AM) is a vasodilatory and chronotropic peptide that has cardioprotective properties in settings of ischemia-reperfusion. Specifically, the 52-amino acid peptide hormone has been shown to reduce infarct size and potentially be protective against malignant arrhythmias. AM reduces L-type calcium currents in rabbit ventricular myocytes, and can possibly be utilized as an antiarrhythmic drug. However, at present it is unclear whether AM has cardiac electrophysiological effects in vivo.
\end{abstract}

In the present study, we investigated whether AM affects sinus and atrioventricular nodal functions, cardiac pacing thresholds, refractory properties and intracardiac conduction intervals in an intact porcine model (Norwegian landrace and Yorkshire hybrids; $n=12$ ). Hemodynamic and electrophysiological parameters were recorded at baseline and following $60 \mathrm{~min}$ of AM-infusion (100 $\mathrm{ng} / \mathrm{kg} / \mathrm{min}$ ).

The hemodynamic effects of AM were characterized by a reduced mean arterial pressure ( $76 \pm 9$ vs. $57 \pm 6$; p<0.05; mmHg), tachycardia (91 \pm 13 vs. $112 \pm 15 ; p<0.05$; beats $/ \mathrm{min})$ and an augmented cardiac index $(131 \pm$ 20 vs. $176 \pm 28 ; p<0.05 ; \mathrm{ml} / \mathrm{min} / \mathrm{kg}$ ). The sinus cycle length was reduced $(674 \pm 89$ vs. $546 \pm 73 ; \mathrm{p}<0.05 ; \mathrm{ms})$, sinoatrial conduction time was unaltered ( $79 \pm 24$ vs. $72 \pm 19$; ns; ms), and the sinus node recovery time (SNRT) was reduced at paced cycle lengths of $425 \mathrm{~ms}, 375 \mathrm{~ms}$ and $325 \mathrm{~ms}$ (average SNRT $1034 \pm 449$ vs.704 $\pm 141 ; p<0.05$; $\mathrm{ms}$ ). Diastolic pacing thresholds, effective refractory periods, Wenckebach cycle length and intracardiac conduction intervals were unaffected by AM.

In conclusion, AM stimulates sinus node function with a reduced sinus cycle length and an increased automaticity, but has no further detectable intracardiac electrophysiological effects. Whether the increased automaticity is a direct effect on the sinus node or an indirect effect mediated through the autonomic nervous system remains to be determined.

Keywords: Adrenomedullin; Electrophysiology; Sinus node function; Sinus node recovery time

\section{Introduction}

Adrenomedullin (AM) is a 52-amino acid peptide with vasodilatory and chronotropic properties in vivo [1]. The peptide reduces myocardial infarct size, limits ischemia-reperfusion injury, and lowers heart-failure mortality in various rodent models [2-4]. The vasodilatory effect is directly on the vasculature, whereas the chronotropic effect is mediated by less clear mechanisms possibly involving the autonomous nervous system $[5,6]$.

In an experimental setup using anesthetized rats, AM exerted a protective effect against ischemia-reperfusion induced arrhythmias, and reduced the number of ventricular ectopic beats and incidence of ventricular tachycardia and fibrillation after coronary ligation [7]. This anti-arrhythmic effect has been suggested to be mediated through nitric oxide and peroxynitrite pathways. However, whether such an effect is related to altered myocardial wall stress, local biochemical effects of $\mathrm{AM}$ or direct electrophysiological effects in the ischemic myocardium, is still undetermined.

There are presently no studies describing cardiac electrophysiological effects of AM in an intact animal model. However, AM has been shown to reduce L-type calcium currents in rabbit ventricular myocytes [8], and therefore can have direct effects on the electrophysiological and electromechanical cross-talk in the myocardium itself. In addition, proadrenomedullin N-terminal 20 protein, coexpressed from the AM-gene, dampens the excitability in adrenergic nerve terminals through a hyperpolarizing effect on an inward rectifying $\mathrm{K}^{+}$current [9].
To further assess the potential electrophysiological effects of $\mathrm{AM}$, we investigated whether AM affects sinus and atrioventricular nodal function, cardiac pacing thresholds, refractory properties and intracardiac conduction intervals in an intact porcine model.

\section{Methods}

The experimental protocol was approved by the local steering committee of the Norwegian Animal Research Authority and was conducted in accordance with the Guide for the Care and Use of Laboratory Animals published by the US National Institutes of Health (NIH Publication No. 85-23, revised 1996). Twelve castrated male domestic pigs (Norwegian Landrace and Yorkshire hybrids) weighing $29 \pm 3 \mathrm{~kg}$ were adapted to the animal facilities for 4-7 days and fasted overnight before the experiment with free access to water.

*Corresponding author: Dr. Truls Myrmel, Cardiovascular Research Group Department of Clinical Medicine, Faculty of Health Sciences, UiT The Arctic University of Norway, NO-9037 Troms $ø$, Norway, Tel: +47 91699167; Fax: +47 77628298; E-mail: truls.myrmel@uit.no; truls.myrmel@unn.no

Received June 10, 2017; Accepted August 07, 2017; Published August 14, 2017

Citation: Stenberg TA, Koren S, Kildal AB, Myrmel T (2017) Adrenomedullin Stimulates Porcine Sinus Node Automaticity. Cardiovasc Pharm Open Access 6 215. doi: $10.4172 / 2329-6607.1000215$

Copyright: (c) 2017 Stenberg TA, et al. This is an open-access article distributed under the terms of the Creative Commons Attribution License, which permits unrestricted use, distribution, and reproduction in any medium, provided the original author and source are credited. 


\section{Anesthesia and instrumentation}

The animals were premedicated with $20 \mathrm{mg} / \mathrm{kg}$ ketamine, $0.5 \mathrm{mg} /$ $\mathrm{kg}$ midazolam and $0.03 \mathrm{mg} / \mathrm{kg}$ atropine administered intramuscularly. General anesthesia was induced with $0.01 \mathrm{mg} / \mathrm{kg}$ fentanyl and $10 \mathrm{mg} /$ $\mathrm{kg}$ sodium pentobarbital through an ear vein and maintained with 0.03 $\mathrm{mg} / \mathrm{kg} / \mathrm{h}$ fentanyl, $5 \mathrm{mg} / \mathrm{kg} / \mathrm{h}$ sodium pentobarbital and $0.4 \mathrm{mg} / \mathrm{kg} / \mathrm{h}$ midazolam through a central venous catheter. The animals were intubated and mechanically ventilated using a volume-controlled ventilator adjusted according to capnography and arterial blood gases with a positive endexpiratory pressure of $5 \mathrm{~cm} \mathrm{H} O$. The circulating volume was maintained by a $1.25 \mathrm{~g} / \mathrm{l}$ glucose-enriched $15 \mathrm{ml} / \mathrm{kg} / \mathrm{h}$ saline infusion. $10000 \mathrm{IU}$ heparin was administered to avoid catheter clotting and thromboembolic episodes. A normal core temperature was maintained at $38^{\circ} \mathrm{C}$ using a thermal mattress. A $10 \mathrm{Fr}$ suprapubic catheter was used to drain the urinary bladder. A series of introducer sheaths were placed in the external jugular veins, right femoral artery and femoral veins to facilitate vascular recordings and interventions as follows:

- A 7 Fr thermodilution catheter through the left external jugular vein to measure central venous pressure, mean pulmonary artery pressure, cardiac index, mixed venous $\mathrm{O}_{2}$ saturation, and core temperature.

- Measurement of mean arterial pressure and arterial blood sampling.

- Cardiac electrophysiological assessment with 6 Fr quadripolar catheters in the high right atrium (HRA) and right ventricle and a 6 Fr decapolar catheter across the His bundle.

\section{Experimental protocol}

Each animal served as its own control. A 1-hour stabilization period preceded baseline hemodynamic and electrophysiological parameter recordings. After baseline measurements $100 \mathrm{ng} / \mathrm{kg} / \mathrm{min}$ AM (human $\mathrm{AM}_{1-52}$, Bachem, Switzerland) was infused for $60 \mathrm{~min}$ and a new set of recordings were obtained. In a subset of the animals, measurements were performed $120 \mathrm{~min}$ after the withdrawal of AM to control for time effects in the model $(n=5)$.

\section{Electrophysiological analysis}

A 12-lead surface ECG and intracardiac electrograms were recorded at a sampling rate of $2000 \mathrm{~Hz}$ using a 128-channel recorder with an integrated stimulator (EP WorkMate, St. Jude Medical, Norway). HRA pacing were performed using leads 1-4, whereas the HRA signal was obtained with leads 2-3. Right ventricular pacing was performed using leads $1-2$, whereas the right ventricular signal was acquired by leads 3-4. The His bundle recording was obtained by leads clearly showing a proximal His bundle signal, and this was further verified by a typical response to atrial rate incremental pacing. When catheter positioning was completed, all catheters remained stationary throughout the experiment to allow detailed comparisons of electrophysiological properties. An initial 10-min recording was obtained to assess spontaneous cycle length and intracardiac conduction intervals. Subsequently, intracardiac conduction intervals and sinus node recovery time (SNRT) was assessed at high right atrial paced cycle lengths of 425,375 and $325 \mathrm{~ms}$ using a square $2 \mathrm{~ms} \mathrm{S1}$ with $2 \mathrm{X}$ diastolic pacing threshold. Sinoatrial conduction time (SACT) was assessed by the method described by Narula et al. [10]. Briefly, 10 square $2 \mathrm{~ms} \mathrm{S1}$ impulses were delivered to the HRA with a cycle length corresponding to 10 beats/min above sinus rhythm. The interval between the last paced atrial signal (Ap) and the first sinus escape atrial signal (A) was measured in triplicate, and SACT was calculated as Ap-A minus A1A1. Wenckebach cycle length was assessed by a stimulus train of 10 square $2 \mathrm{~ms} \mathrm{S1}$ impulses to the HRA and by decreasing S1S1 until atrioventricular nodal block and the Wenckebach phenomenon was observed. Atrial, atrioventricular nodal, His-Purkinje and right ventricular effective refractory periods were determined using a stimulus train of 10 square $2 \mathrm{~ms} \mathrm{S1}$, and with $10 \mathrm{~ms}$ decrements in S1S2 until refractoriness was encountered.

\section{Statistics}

Hemodynamic and electrophysiological data are presented as mean \pm SD. The data were analyzed using a linear mixed-models approach with a restricted maximum likelihood method and the subject identifier as the random effect. P-values were adjusted for multiple comparisons using Tukey's test. P-values $<0.05$ were regarded as statistically significant, and all analyses were conducted in JMP 13.0 (SAS Institute).

\section{Results}

All 12 animals were included in the analyses. We observed a potent vasodilatory and chronotropic effect of AM (Figure 1 and Table 1).
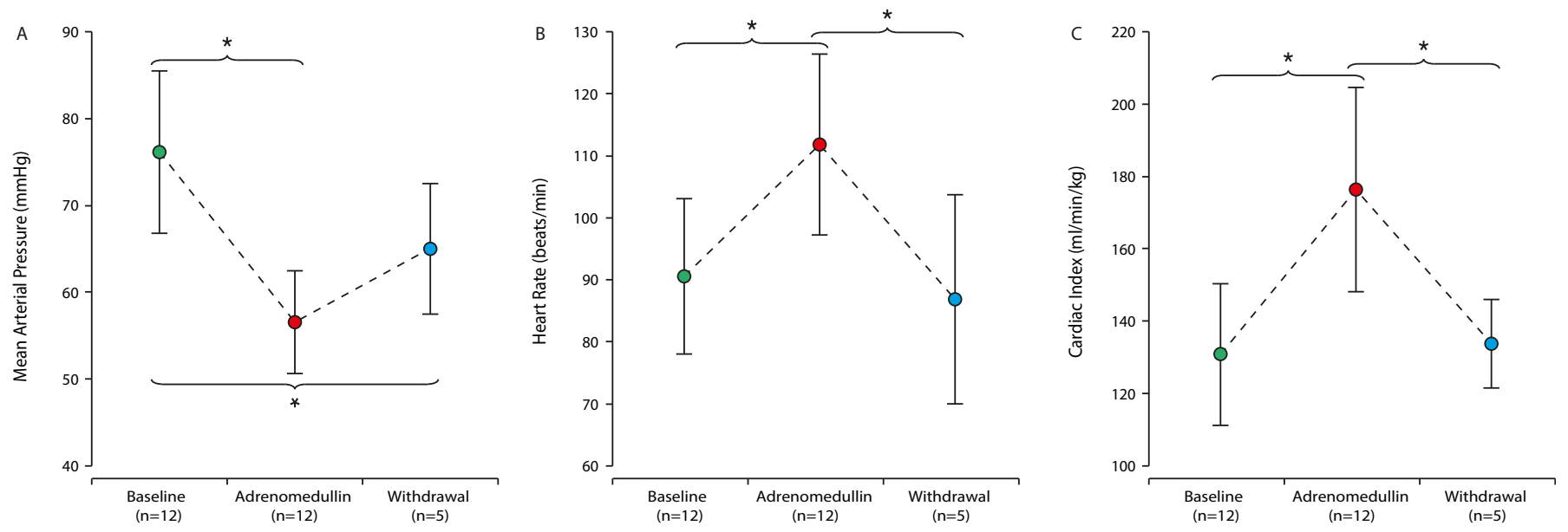

Figure 1: The hemodynamic effects of adrenomedullin (AM) were characterized by hypotension (panel A), tachycardia (panel B) and an augmented cardiac minute volume (panel $\mathrm{C}$ ). Mean arterial pressure demonstrated a partial return to baseline values upon withdrawal of $\mathrm{AM}$, and heart rate and cardiac minute volume returned to baseline, ${ }^{*} p<0.05$ 
Citation: Stenberg TA, Koren S, Kildal AB, Myrmel T (2017) Adrenomedullin Stimulates Porcine Sinus Node Automaticity. Cardiovasc Pharm Open Access 6: 215. doi: 10.4172/2329-6607.1000215

Page 3 of 5

Mean arterial, systolic and diastolic blood pressures were reduced, and a slight decrease in mean pulmonary artery pressure was observed (all $\mathrm{p}<0.05)$. Heart rate and cardiac index increased significantly $(\mathrm{p}<0.05)$. Upon withdrawal of AM hemodynamic parameters returned to baseline values, however mean arterial pressure was slightly reduced at withdrawal compared to baseline. The core temperature remained constant throughout the experiments. Arterial blood potassium $(3.9 \pm$ 0.4 vs. $4.0 \pm 0.4 ; \mathrm{ns} ; \mathrm{mmol} / \mathrm{l})$ and free calcium ( $1.0 \pm 0.2$ vs. $1.0 \pm 0.2$; $\mathrm{ns} ; \mathrm{mmol} / \mathrm{l})$ levels were also consistent throughout the study protocol.

AM induced a significant reduction in sinus cycle length $(674 \pm$ 89 vs. $546 \pm 73 ; \mathrm{ms} ; \mathrm{p}<0.05)$. Sinus node automaticity increased as reflected by reduced SNRT at paced cycle lengths of $425 \mathrm{~ms}$ (997 \pm 335 vs. $707 \pm 165 ; \mathrm{ms} ; \mathrm{p}<0.05), 375 \mathrm{~ms}(1063 \pm 482$ vs. $718 \pm 144 ; \mathrm{ms}$; $\mathrm{p}<0.05)$ and $325 \mathrm{~ms}(1041 \pm 568$ vs. $688 \pm 118 ; \mathrm{ms} ; \mathrm{p}<0.05)$. Upon withdrawal of AM, these values returned significantly toward baseline with the exception of SNRT when paced at a cycle length of $325 \mathrm{~ms}$. Average sinus cycle length and SNRT are presented as $n=1$ for all animals in Figure 2. Furthermore, an example measurement of SNRT from an animal demonstrating large alteration in SNRT is shown in Figure 2. SNRT values were also analyzed excluding this animal with similar results $(925 \pm 258$ vs. $704 \pm 148$; ms; $\mathrm{p}<0.05)$.

SACT was unaffected by AM (79 \pm 24 vs. $72 \pm 19$; ns; ms). Wenckebach cycle length was unaltered (206 \pm 34 vs. $210 \pm 24$; ns; ms). Diastolic pacing thresholds, effective refractory periods and intracardiac conduction intervals were unaffected by AM (Tables 2 and 3). With respect to conduction velocity, there were significant reductions of the $\mathrm{RR}$ and QT intervals along with the corrected QT interval. This was due to AM's chronotropic effect as it was abolished during paced cycle lengths. An example trace demonstrating intracardiac conduction interval measurements is shown in Figure 3.

\begin{tabular}{|c|c|c|c|c|c|c|c|c|c|c|}
\hline & MAP & SYS & DIA & CVP & MPAP & PAOP & CI & SV & HR & TEMP \\
\hline BL & $76 \pm 9$ & $110 \pm 12$ & $56 \pm 9$ & $8 \pm 2$ & $19 \pm 2$ & $7 \pm 4$ & $131 \pm 20$ & $43 \pm 6$ & $91 \pm 13$ & $37.7 \pm 0.6$ \\
\hline AM & $57 \pm 8^{*}$ & $87 \pm 8^{*}$ & $38 \pm 8^{*}$ & $8 \pm 1$ & $18 \pm 2^{*}$ & $8 \pm 6$ & $176 \pm 12^{*}$ & $46 \pm 3$ & $112 \pm 17^{*}$ & $37.7 \pm 0.2$ \\
\hline WD & $65 \pm 8^{*}$ & $102 \pm 8^{\#}$ & $46 \pm 8^{*}$ & $8 \pm 1$ & $20 \pm 2^{*}$ & $4 \pm 6$ & $134 \pm 12^{\#}$ & $44 \pm 3$ & $87 \pm 17^{\#}$ & $37.8 \pm 0.2$ \\
\hline
\end{tabular}

Hemodynamic parameters at baseline (BL; $n=12)$, following $60 \mathrm{~min}$ of $100 \mathrm{ng} / \mathrm{kg} / \mathrm{min}$ adrenomedullin infusion (AM; $\mathrm{n}=12)$ and $120 \mathrm{~min}$ after withdrawal (WD; $=5$ ). AM has a hypotensive and chronotropic effect, and also augments cardiac output primarily through frequency-dependent mechanisms as stroke volume only tended to increase in this model. MAP: Mean Arterial Pressure (mmHg); SYS: Systolic arterial pressure (mmHg); DIA: Diastolic arterial pressure (mmHg); CVP: Central Venous Pressure (mmHg); MPAP: Mean Pulmonary Artery Pressure (mmHg); PAOP: Pulmonary Artery Occlusion Pressure (mmHg); Cl: Cardiac Index (ml/min/kg); SV: Stroke Volume (ml); HR:

Heart Rate (beats/min); TEMP: Temperature $\left({ }^{\circ} \mathrm{C}\right),{ }^{*} \mathrm{p}<0.05$ vs. baseline, ${ }^{*} \mathrm{p}<0.05$ vs. AM.

Table 1: Hemodynamics.
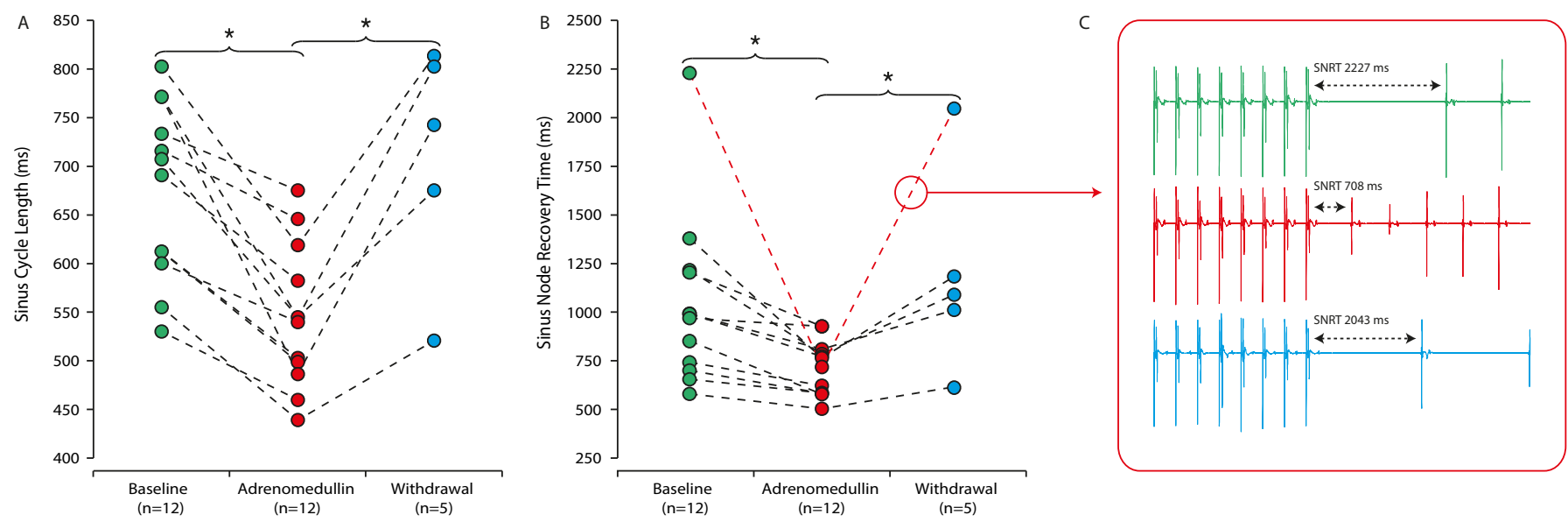

Figure 2: The electrophysiological assessment demonstrated that adrenomedullin (AM) stimulated sinus node function as evident by a reduced sinus cycle length (panel A) and a reduced SNRT (panels B-C). Average sinus cycle length and SNRT are presented as $n=1$ for all animals, and demonstrate a clear and consistent trend within each animal. When AM is withdrawn these parameters return to baseline values. Furthermore, an example of SNRT assessment in an animal demonstrating a substantial decrease in SNRT is shown in panel C, ${ }^{*} p<0.05$

\begin{tabular}{|c|c|c|c|c|c|c|c|c|c|}
\hline & \multicolumn{3}{|c|}{$\mathbf{4 2 5} \mathbf{~ m s}$} & \multicolumn{3}{c|}{$\mathbf{3 7 5} \mathbf{~ m s}$} & \multicolumn{3}{c|}{$\mathbf{3 2 5} \mathbf{~ m s}$} \\
\cline { 2 - 8 } & $\mathbf{B L}$ & AM & WD & BL & AM & WD & BL & AM & WD \\
\hline aDPT & $0.9 \pm 0.3$ & $0.8 \pm 0.3$ & $0.9 \pm 0.3$ & $0.9 \pm 0.4$ & $0.9 \pm 0.4$ & $0.9 \pm 0.3$ & $1.0 \pm 0.5$ & $0.9 \pm 0.4$ & $1.0 \pm 0.2$ \\
\hline rvDPT & $0.5 \pm 0.2$ & $0.5 \pm 0.1$ & $0.7 \pm 0.2^{* \#}$ & $0.5 \pm 0.2$ & $0.5 \pm 0.2$ & $0.8 \pm 0.3^{* \#}$ & $0.4 \pm 0.2$ & $0.5 \pm 0.2$ & $0.7 \pm 0.3^{* \#}$ \\
\hline aERP & $177 \pm 21$ & $178 \pm 24$ & $192 \pm 43$ & $176 \pm 20$ & $176 \pm 25$ & $194 \pm 50$ & $166 \pm 14$ & $165 \pm 21$ & $178 \pm 24^{* \#}$ \\
\hline avERP & $227 \pm 37$ & $220 \pm 28$ & $210 \pm 35$ & $211 \pm 33$ & $206 \pm 29$ & $213 \pm 49$ & $187 \pm 21$ & $188 \pm 24$ & $187 \pm 15$ \\
\hline hpERP & $255 \pm 17$ & $246 \pm 25$ & $248 \pm 40$ & $232 \pm 26$ & $222 \pm 27$ & $230 \pm 36$ & $203 \pm 20$ & $199 \pm 24$ & $193 \pm 21$ \\
\hline rvERP & $249 \pm 23$ & $249 \pm 20$ & $254 \pm 18$ & $239 \pm 16$ & $238 \pm 16$ & $246 \pm 19$ & $223 \pm 14$ & $223 \pm 14$ & $234 \pm 19^{\#}$ \\
\hline
\end{tabular}

Electrophysiological parameters at baseline (BL; $n=12)$, following $60 \mathrm{~min}$ of $100 \mathrm{ng} / \mathrm{kg} / \mathrm{min}$ adrenomedullin infusion $(A M ; n=12)$ and $120 \mathrm{~min}$ after withdrawal (WD; $n=5)$. $A M$ was electrophysiologically neutral with respect to high right atrial and right ventricular diastolic pacing thresholds using cycle lengths of 425,375 and 325 ms. Furthermore, AM demonstrated no effect on effective refractory properties in the cardiac conduction system. aDPT: Atrial Diastolic Pacing Threshold (mA); rvDPT: Right Ventricular Diastolic Pacing Threshold (mA); aERP: Atrial Effective Refractory Period (ms); avERP: Atrioventricular Nodal Effective Refractory Period (ms); hpERP: His-Purkinje Effective Refractory Period (ms); rvERP: Right Ventricular Effective Refractory Period (ms), ${ }^{*} \mathrm{p}<0.05$ vs. baseline, ${ }^{*} \mathrm{p}<0.05$ vs. AM.

Table 2: Diastolic pacing thresholds and effective refractory periods. 
Citation: Stenberg TA, Koren S, Kildal AB, Myrmel T (2017) Adrenomedullin Stimulates Porcine Sinus Node Automaticity. Cardiovasc Pharm Open Access 6: 215. doi: 10.4172/2329-6607.1000215

Page 4 of 5

\begin{tabular}{|c|c|c|c|c|c|c|c|c|c|c|c|c|}
\hline & \multicolumn{3}{|c|}{ Sinus Rhythm } & \multicolumn{3}{|c|}{$425 \mathrm{~ms}$} & \multicolumn{3}{|c|}{$375 \mathrm{~ms}$} & \multicolumn{3}{|c|}{$325 \mathrm{~ms}$} \\
\hline & $B L$ & AM & WD & BL & AM & WD & BL & AM & WD & BL & AM & WD \\
\hline $\mathbf{P Q}$ & $118 \pm 15$ & $110 \pm 15$ & $125 \pm 9$ & $158 \pm 23$ & $155 \pm 23$ & $192 \pm 30^{* \#}$ & $162 \pm 22$ & $156 \pm 23$ & $188 \pm 23^{* \#}$ & $158 \pm 19$ & $154 \pm 19$ & $181 \pm 16^{* \#}$ \\
\hline QRS & $51 \pm 3$ & $51 \pm 3$ & $51 \pm 2$ & $50 \pm 2$ & $50 \pm 3$ & $52 \pm 3^{* \#}$ & $50 \pm 3$ & $49 \pm 2$ & $51 \pm 2$ & $49 \pm 1$ & $49 \pm 2$ & $51 \pm 2$ \\
\hline RR & $672 \pm 92$ & $546 \pm 74^{*}$ & $705 \pm 110^{\#}$ & $424 \pm 4$ & $422 \pm 7$ & $431 \pm 8^{* \#}$ & $373 \pm 5$ & $376 \pm 3$ & $373 \pm 8$ & $326 \pm 3$ & $316 \pm 25$ & $327 \pm 2$ \\
\hline QT & $344 \pm 28$ & $324 \pm 27^{\star}$ & $345 \pm 26^{\#}$ & $298 \pm 12$ & $294 \pm 9$ & $286 \pm 13$ & $277 \pm 12$ & $273 \pm 10$ & $273 \pm 10$ & $255 \pm 11$ & $253 \pm 15$ & $264 \pm 17$ \\
\hline QTc & $421 \pm 33$ & $439 \pm 23^{*}$ & $413 \pm 18$ & $458 \pm 19$ & $452 \pm 13$ & $436 \pm 17$ & $454 \pm 21$ & $446 \pm 15$ & $446 \pm 21$ & $446 \pm 19$ & $451 \pm 32$ & $461 \pm 28$ \\
\hline $\mathbf{A H}$ & $69 \pm 9$ & $67 \pm 8$ & $71 \pm 12$ & $79 \pm 14$ & $83 \pm 15$ & $104 \pm 27^{\#}$ & $87 \pm 17$ & $84 \pm 16$ & $102 \pm 21^{* \#}$ & $83 \pm 16$ & $81 \pm 15$ & $96 \pm 13^{* \#}$ \\
\hline HV & $24 \pm 5$ & $24 \pm 5$ & $32 \pm 6$ & $25 \pm 5$ & $25 \pm 5$ & $37 \pm 5^{\star \#}$ & $25 \pm 5$ & $26 \pm 5$ & $35 \pm 5^{\#}$ & $26 \pm 4$ & $27 \pm 4$ & $34 \pm 4^{* \#}$ \\
\hline AV & $93 \pm 13$ & $89 \pm 10$ & $103 \pm 10^{\#}$ & $110 \pm 22$ & $106 \pm 18$ & $141 \pm 24^{* \#}$ & $116 \pm 19$ & $108 \pm 17$ & $137 \pm 18^{* \#}$ & $112 \pm 16$ & $106 \pm 15$ & $130 \pm 11^{* \#}$ \\
\hline
\end{tabular}

Electrophysiological parameters at baseline (BL; $n=12$ ), following $60 \mathrm{~min}$ of $100 \mathrm{ng} / \mathrm{kg} / \mathrm{min}$ adrenomedullin infusion (AM; $n=12)$ and 120 min after withdrawal (WD; $n=5$ ). Conduction intervals derived from the surface ECG demonstrated a frequency-dependent decrease in the RR, QT and corrected QT (QTc) intervals during sinus rhythm. The observed interval reductions were abolished during high right atrial pacing using cycle lengths of 425,375 and 325 ms. AM had no discernible effect on intracardiac conduction intervals. PQ, QRS, RR, QT and QTc intervals (ms) are derived from the surface ECG. AH, HV and AV (AH + HV) intervals (ms) are measured from the intracardiac electrograms (Figure 3 ), ${ }^{*} p<0.05$ vs. baseline, ${ }^{*} p<0.05$ vs. AM.

Table 3: Cardiac conduction intervals.

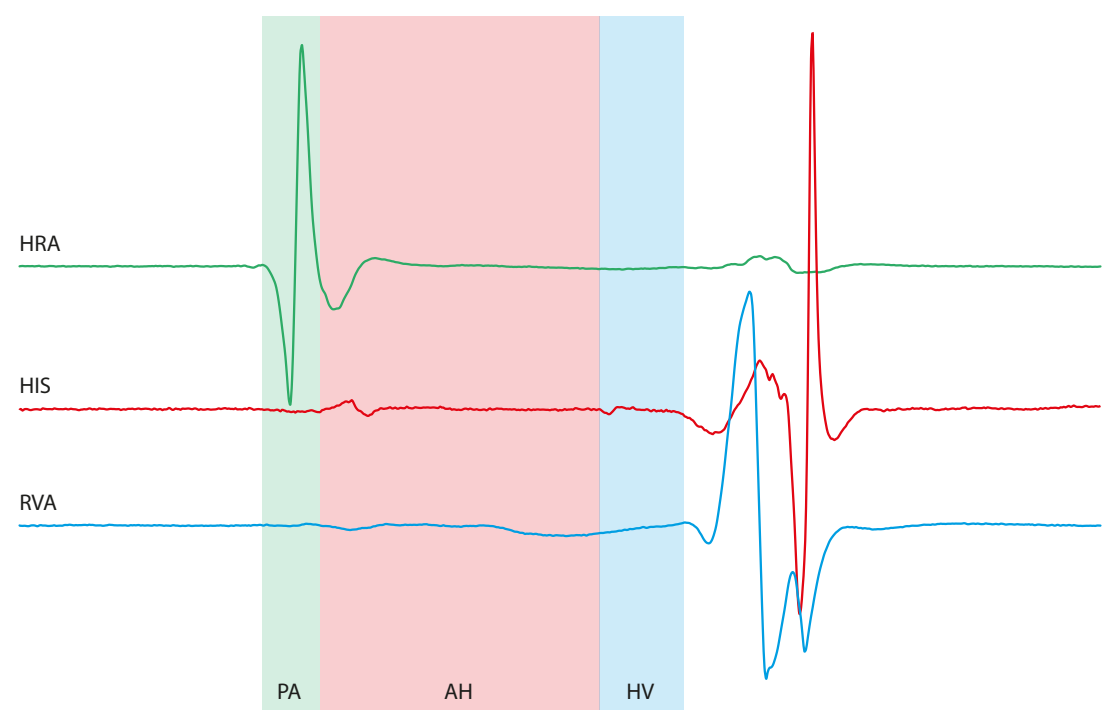

Figure 3: Intracardiac electrograms from the High Right Atrium (HRA), His bundle area (HIS) and Right Ventricular Apex (RVA) demonstrating measurement of intracardiac conduction intervals.

\section{Discussion}

The main observation in this study was that AM alters sinus node function with a reduced sinus cycle length and an increased potential automaticity as evident by the reduced SNRT. Furthermore, we found that atrial, atrioventricular nodal and ventricular pacing thresholds and refractory properties are unaltered by the hormone. The PR, RR and QT intervals from the electrocardiograms were all reduced during AM-infusion, but during paced cycle lengths intracardiac conduction intervals were unaffected by AM. Thus, AM exerts a chronotropic effect through mechanisms that alter sinus node automaticity, and it exerts frequency dependent changes in cardiac conduction times. Importantly, AM had no directly discernible effects on intracardiac conduction in a pharmacological dose with potentially desirable hemodynamic effects.

Whether the effect of AM on sinus node function is caused by a direct effect on the membranes and ion-currents in the sinus node, or mediated indirectly by autonomic reflex pathways, is presently not clear. The peptide has potent vasodilatory properties, and reduces arterial blood pressure with parallel reflectory increases in heart rate and cardiac output [11]. Importantly, the chronotropic effect is prevented by autonomic ganglion blockade, and the hypotensive effect of AM is more pronounced during autonomic ganglion blockade [6].
Furthermore, AM has shown the ability to act directly on the neurons in area postrema [12] and then, through autonomic reflexes, result in a rise in blood pressure and heart rate in this particular setup [13]. The effect of proadrenomedullin $\mathrm{N}$-terminal 20 peptide, acting as a modulator of ganglionic excitability, point to a possible interacting effect of these two adrenomedullin on the autonomic control of the heart rate $[9,14]$.

AM infusion has been demonstrated to augment cardiac sympathetic nerve activity in conscious sheep [5], indicating that the chronotropic effect of AM is mediated by autonomic reflexes. Cardiac output and stroke volume both increase during AM infusion and autonomic ganglion blockade [6]. However, this may be explained by the peptides load-altering properties with a reduced afterload and a relatively sustained venous return, in addition to a vasodilatation of the coronary arteries and increased coronary flow due to increased production of nitric oxide [15]. We have previously demonstrated that low-dose AM augments the inotropic effect of epinephrine, and an increased sympathetic drive during AM treatment can possibly be the mechanism behind such augmentation [16].

$\mathrm{AM}$ has acted anti-arrhythmic with a remarkable reduction in ventricular ectopic beats and occurrence of ventricular fibrillation when given before experimental coronary occlusion. Existing evidence indicate that this effect is mediated through nitric oxide 
Citation: Stenberg TA, Koren S, Kildal AB, Myrmel T (2017) Adrenomedullin Stimulates Porcine Sinus Node Automaticity. Cardiovasc Pharm Open Access 6: 215. doi: 10.4172/2329-6607.1000215

and peroxynitrite [7]. However, this study did not explore potential reductions in the extent of myocardial infarctions or a reduced wall stress induced by AM. Thus, whether AM protects the ischemic myocardium through a direct electrophysiological effect or via a reduced mechanical tension is still uncertain. AM partially inhibits progression of left ventricular remodeling when given as a long-term infusion ( 4 weeks) immediately after ligation of the left coronary artery, and do also lower the left ventricular end-diastolic pressure compared to controls [17]. Such mechanical unloading might be beneficial also by reducing stretch-induced ischemic arrhythmias.

AM has a cardioprotective effect when given during reperfusion after ischemia by reducing myocardial infarct size, left ventricular end diastolic pressure and myocardial apoptotic cell death, partly due to an increase in PI3K/AKT [4]. Thus, the protective effect of long-term treatment with AM can potentially be related to the anti-apoptotic effect of AM set off by reperfusion. To what extent such tissue effects can have secondary stabilizing effects on electrophysiological properties in the myocardium is presently unknown.

\section{Conclusion}

The results show that $\mathrm{AM}$ influences sinus node function with a reduced sinus cycle length and an increased potential automaticity as evident by the reduced SNRT.

\section{Acknowledgment}

This study was supported in part by the Simon Fougner Hartmann research foundation and the Health Authorities of North Norway.

\section{References}

1. Ishimitsu T, Ono H, Minami J, Matsuoka H (2006) Pathophysiologic and therapeutic implications of adrenomedullin in cardiovascular disorders. Pharmacol Ther 111: 909-927.

2. Nakamura R, Kato J, Kitamura K, Onitsuka H, Imamura T, et al. (2004) Adrenomedullin administration immediately after myocardial infarction ameliorates progression of heart failure in rats. Circulation 110: 426-431.

3. Nishida H, Sato T, Miyazaki M, Nakaya H (2008) Infarct size limitation by adrenomedullin: protein kinase A but not PI3-kinase is linked to mitochondrial KCa channels. Cardiovasc Res 77: 398-405.
4. Okumura H, Nagaya N, Itoh T, Okano I, Hino J, et al. (2004) Adrenomedullin infusion attenuates myocardial ischemia/reperfusion injury through the phosphatidylinositol 3-kinase/Akt-dependent pathway. Circulation 109: 242-248.

5. Charles CJ, Jardine DL, Nicholls MG, Richards AM (2005) Adrenomedullin increases cardiac sympathetic nerve activity in normal conscious sheep. J Endocrinol 187: 275-281.

6. Parkes DG, May CN (1997) Direct cardiac and vascular actions of adrenomedullin in conscious sheep. Br J Pharmacol 120: 1179-1185.

7. Looi YH, Kane KA, Mcphaden AR, Wainwright CL (2006) Adrenomedullin acts via nitric oxide and peroxynitrite to protect against myocardial ischaemiainduced arrhythmias in anaesthetized rats. Br J Pharmacol 148: 599-609.

8. Ikenouchi H, Kangawa K, Matsuo H, Hirata Y (1997) Negative inotropic effect of adrenomedullin in isolated adult rabbit cardiac ventricular myocytes. Circulation 95: $2318-2324$.

9. Takano K, Fujita $\mathrm{T}$ (1999) Proadrenomedullin N-terminal 20 peptide hyperpolarizes the membrane by activating an inwardly rectifying $\mathrm{K}+$ current in differentiated PC12 cells. Circ Res 84: 445-450.

10. Narula OS, Shantha N, Vasquez M, Towne WD, Linhart JW (1978) A new method for measurement of sinoatrial conduction time. Circulation 58: 706-714

11. Parkes DG (1995) Cardiovascular actions of adrenomedullin in conscious sheep. Am J Physiol 268: H2574-H2578.

12. Allen MA, Ferguson AV (1996) In vitro recordings from area postrema neurons demonstrate responsiveness to adrenomedullin. Am J Physiol 270: R920-R925.

13. Allen MA, Smith PM, Ferguson AV (1997) Adrenomedullin microinjection into the area postrema increases blood pressure. Am J Physiol 272: R1698-R1703.

14. Takano K, Yamashita N, Fujita T (1996) Proadrenomedullin NH2-terminal 20 peptide inhibits the voltage-gated $\mathrm{Ca}^{2+}$ channel current through a pertussis toxin-sensitive $\mathrm{G}$ protein in rat pheochromocytoma-derived PC 12 cells. J Clin Invest 98: 14-17

15. De Matteo R, May CN (2003) Direct coronary vasodilator action of adrenomedullin is mediated by nitric oxide. $\mathrm{Br} \mathrm{J}$ Pharmacol 140: 1414-1420.

16. Stenberg TA, Kildal AB, How OJ, Myrmel T (2012) Adrenomedullinepinephrine cotreatment enhances cardiac output and left ventricular function by energetically neutral mechanisms. Am J Physiol Heart Circ Physiol 302: $\mathrm{H} 1584-\mathrm{H} 1590$.

17. Nakamura R, Kato J, Kitamura K, Onitsuka H, Imamura T, et al. (2002) Beneficial effects of adrenomedullin on left ventricular remodeling after myocardial infarction in rats. Cardiovasc Res 56: 373-380. 